\title{
Identification of in vitro and in vivo potential metabolites of novel cardiovascular and adrenolytic drugs by liquid chromatography-mass spectrometry with the aid of experimental design
}

\author{
Malgorzata Szultka-Mlynska ${ }^{1, \bigotimes}$, Katarzyna Pauter $^{1,2}$ and Boguslaw Buszewski1 ${ }^{1,2}$ \\ ${ }^{1}$ Department of Environmental Chemistry and Bioanalytics, Faculty of Chemistry, Nicolaus Copernicus University, \\ Gagarina 7, 87100 Torun, Poland \\ ${ }^{2}$ Centre for Modern Interdisciplinary Technologies, Nicolaus Copernicus University, Wilenska 4, 87100 Torun, Poland
}

\section{Article info}

Article history:

Received: $13^{\text {th }}$ October 2019

Accepted: $26^{\text {th }}$ November 2019

\section{Keywords:}

Adrenolytic drugs

Cardiovascular drugs

High performance liquid chromatography

Liver microsomes

Mass spectrometry

\begin{abstract}
Drug metabolism in liver microsomes was studied in vitro using liquid chromatography-tandem mass spectrometry (LC-MS/MS). Relevant drug was incubated with dog, human and rat liver microsomes (DLMs, HLMs, RLMs) along with NADPH, and the reaction mixture was analyzed by LC-MS/MS to obtain specific metabolic profile. GRACE analytical C18 column, Vision HT $(50 \times 2 \mathrm{~mm}$, $1.5 \mu \mathrm{m})$ was implemented with acetonitrile and water $(+5 \mathrm{mM}$ ammonium acetate) in a gradient mode as the mobile phase at a flow $0.4 \mathrm{~mL} \cdot \mathrm{min}^{-1}$. Different phase I and phase II metabolites were detected and structurally described. The metabolism of the studied drugs occurred via oxidation, hydroxylation and oxidative deamination processes. Conjugates with the glucuronic acid and sulfate were also observed as phase II biotransformation. The central composite design (CCD) showed that factors, such as time incubation, liver microsomal enzymes concentration and NADPH concentration, along with drying gas temperature, nebulizer gas pressure and capillary voltage significantly affected the final response of the method. This study describes the novel information about the chemical structure of the potential metabolites of selected biologically active compounds, which provide vital data for further pharmacokinetic and in vivo metabolism studies.
\end{abstract}

(C) University of SS. Cyril and Methodius in Trnava

\section{Introduction}

In recent years there has been a growing interest in research which improves our understanding of pathological phenomena responsible for the development and progression of various illnesses. With new diagnostic tools, the background of the most complex anomalies can be discovered (Li 2001; Bussiere 2008). Huge progress has also been made in the therapy of many illnesses, which measurably translates into the extended overall survival of the patient (Wrighton et al. 1995; Jia et al. 2007; Rostami-Hodjegan et al. 2007).
Furthermore, as chromatographic techniques have been developed and combined with sensitive detection methods, the use of metabolomics has increased in the recent years (Goldman et al. 2004; Persell 2011). This made it possible to determine and identify substances in biological samples with the view to clinical diagnosing of illnesses and their stages as biomarkers of various human diseases have been discovered (Ingelman-Sundberg 1999, 2004; Zisaki et al. 2015). An important trend in a pharmaceutical analysis is determining metabolic profiles after administering the drug to investigate what happens to it in the organism. 
Currently the most popular technique in the analysis of drugs and their metabolites is high-performance liquid chromatography combined with mass spectrometry. This combination is used most often because of its very low limit of detection, good selectivity, accuracy and the precision of quantification. The information obtained can be used to identify and quantitatively determine the studied compounds (Xu et al. 2007; Lietz et al. 2013).

The determination of metabolites requires the use of multidimensional separation techniques. Therefore the LC/MS analysis of selected drugs and their metabolites is to evaluate their concentration in real time (Wicha et al. 2018; Zhang et al. 2018). There are drug metabolites whose structures may resemble the structure of the parent drug to a lesser or greater degree and which also show a pharmaceutical activity resembling that of the parent drug; this creates a significant problem in therapeutic drug monitoring (TDM) (Kang et al. 2009). Monitored therapy is a useful tool in the process of personalizing treatments with drugs of varied pharmacological activity, because drug pharmacokinetics can differ significantly from patient to patient, which results from the influence of numerous interactions, comorbidities, genetic polymorphism or patients failing to adhere to the prescribed dose schedule (Guengerich 2000). The monitoring of drug concentrations in blood is widely used in selected clinical specializations, e.g. neurology, cardiology and psychiatry. The most often monitored types of drugs include antibiotics, anti-seizure medicines, anti-arrhythmic medicines, painkillers, immunosuppressant substances, anti-cancer drugs and tricyclic antidepressants (Cholerton et al. 1992; Nicholson et al. 1999; Yan et al. 2001).

The enzyme family of cytochromes P450 are membrane proteins present mostly in the microsomes of endoplasmic reticulum and in membrane structures of mitochondrion (Lamb et al. 2007). Microsomes are closed vesicles in the membrane of endoplasmic reticulum in liver cells (coming from mice, rats and humans) which contain a number of phase I and II metabolic enzymes, including the monooxygenase enzymatic complex that comprises cytochrome $\mathrm{P} 450$ and its NADPH-dependent reductase (Isin et al. 2007).
Among the numerous microsomal fraction enzymes in liver cells, the ones that are most involved in biotransformation reactions of exogenous substances are isoenzymes of cytochrome P450. There have been discovered more than a dozen of cytochrome $\mathrm{P} 450$ isoenzymes that participate in the oxidation of many drugs used in medicine. Each isoenzyme is coded by a different gene (Degtyarenko et al. 1993). The so-called gene superfamily that codes the various isoenzymes of cytochrome $\mathrm{P} 450$ has been classified into families and sub-families depending on the degree of similarities in the amino acid sequence of particular isoenzymes. Approximately $95 \%$ of the currently used medicines are metabolized by this enzymes group (Houston 1994; Wiekers et al. 2005; Sahi et al. 2010).

Here we report the successful application of the optimized procedure and analysis method to determine and identify the selected cardiovascular and adrenolytic drugs (aliskiren, metoprolol, mexiletine, oxprenolol, prasugrel, propafenone, propranolol and rivaroxaban) and their potential metabolites found in different microsomal liver fractions (dog, human and rat). Conventional triple quadrupole mass spectrometer (MS/MS) are the most popular analytical tools due to its excellent quantitative sensitivity, stability and low cost. However, it is not suitable for drug metabolite profiling since their full-scan sensitivity is rather poor. To overcome this problem, a set of predicted precursor-product ion pairs instead of full-scan mode to search for the potential metabolites was used. To the best of our knowledge, no detailed information regarding the structure of their potential metabolites via in vitro studies has been published. Transformations were investigated in the presence of NADPH/UDPGA-P450 oxidoreductase. Central composite face-centered design with fractional factorial design was used in order to evaluate the significance of selected factors. In addition, the composite design helped us to find the optimal values for each factor using the response surface method. Additionally, the results obtained were compared to in vivo experiments, by analyzing plasma samples from patients differing in the level of cytochrome P450 isoenzymes after relevant cardiovascular or adrenolytic drug delivery. 


\section{Experimental}

\section{Chemicals and materials}

Aliskiren (ALI), metoprolol (MET), mexiletine (MEX), oxprenolol (OXP), prasugrel (PRA), propafenone (PRF), propranolol (PRO) and rivaroxaban (RIV) were obtained from SigmaAldrich (Schelldorf, Germany). Glucose-6phosphate, $\quad \beta$-NADP ${ }^{+}, \quad$ glucose-6-phosphate dehydrogenase and uridine 5'-diphosphoglucuronic acid triammonium salt (UDPGA) were obtained from Sigma-Aldrich (Schnelldorf, Germany). Other chemicals of analytical quality (acetonitrile, formic acid, ammonium acetate) were purchased from Merck (Darmstadt, Germany). Microsomes derived from dog, human and rat liver cells (DLMs, HLMs, RLMs) were used in enzymatic studies. The microsomal fractions were stored at $-80{ }^{\circ} \mathrm{C}$ and thawed immediately before each experiment. The water was obtained by means of Milli-Q RG apparatus by Millipore (Millipore Intertech, Bedford, MA, USA) in our laboratory. Assay incubation was done in the thermomixer (Eppendorf, Hamburg, Germany). The evaporation of samples was done on a ScanVac speed vacuum concentrator (Kansas City, USA). Drug-free plasma samples were kindly provided by Nicolaus Copernicus University, Collegium Medicum, Torun (Poland) upon the permission of the Bioethical Commission (no. 585/2017).

\section{Microsomal incubations}

The research on discovering the mechanism of metabolic transformations of selected bioactive compounds was carried out in model conditions with their standard solutions in the presence of microsomal enzyme fraction (coming from dog, human and rat). The incubation of the selected drugs with liver cell microsomal fraction enzymes was to be performed in appropriate reaction buffer (PBS, $\mathrm{pH}$ 7.4). After initial pre-incubation in a heated bath $\left(37^{\circ} \mathrm{C}\right)$, enzymatic reaction cofactor NADPH/UDPGA was added to the mixture of the particular bioactive compound and microsome (enzyme). The obtained reaction mixture was subjected to further incubation at the same temperature for a specific period $(15$,
25 and $35 \mathrm{~min}$ ). Then the resulting solution was cooled in ice bath and an equivalent amount of cooled methyl alcohol was added to stop the reaction. This mixture was centrifuged (5 $15 \mathrm{~min} ; 5,000 \mathrm{rpm})$. The resulting supernatant was analyzed by RP-HPLC with ESI-MS/MS.

Glucuronidation was studied by the incubation of the model drug with DLMs, HLMs and RLMs. The test and blank samples were prepared analogously to phase I metabolism samples, the exception being that $10 \mu \mathrm{l}$ of PBS contained the UDPGA co-factor which mediated the relevant reaction. Beside the above mentioned batches, incubations without NADPH, microsomal protein, target compound, respectively, were analyzed as negative controls.

\section{High performance liquid chromatography tandem mass spectrometry instrumentation and analytical conditions}

The HPLC system consisted of an Agilent 1100 series (Agilent Technologies, Waldbronn, Germany) autosampler, binary pump, degasser and column compartment. Chromatograph separation was performed on a GRACE analytical C18 column, Vision HT $(50 \times 2 \mathrm{~mm}, 1.5 \mu \mathrm{m})$, which was protected by a HPLC Guard $(2.1 \times$ $5 \mathrm{~mm}, 1.8 \mu \mathrm{m})$ at $21^{\circ} \mathrm{C}$. The column was operated gradiently at a flow rate of $0.4 \mathrm{~mL} \cdot \mathrm{min}^{-1}$ with mobile phase composed of $0.1 \%$ ammonium acetate $(\mathrm{A})$ and acetonitrile $(\mathrm{B}): 0-2 \mathrm{~min}, 15 \% \mathrm{~B}$; $2-5$ min, linear to $95 \% \mathrm{~B} ; 5-10 \mathrm{~min}, 95 \% \mathrm{~B}$; and $10-12 \mathrm{~min}$, return to initial conditions. The stop time was $15 \mathrm{~min}$, followed by a $3 \mathrm{~min}$ post-time. The injection volume was $10 \mu 1$.

A 6410 triple quadrupole LC/MS (Agilent Technologies, Waldbronn, Germany) equipped with electrospray ionization (ESI) source was controlled by Mass Hunter workstation data acquisition software and Qualitative Analysis software (version B.01.00). The source temperature was maintained at $290-350{ }^{\circ} \mathrm{C}$, and the ionization voltage was set at 3,500-4,500 V. The nebulizer gas was set at $35-45$ psi. The detection of the ions was performed in the multiple reaction monitoring (MRM) mode. Optimization of the compounddependent parameters were performed by the direct infusion of a standard solution into the ion source 


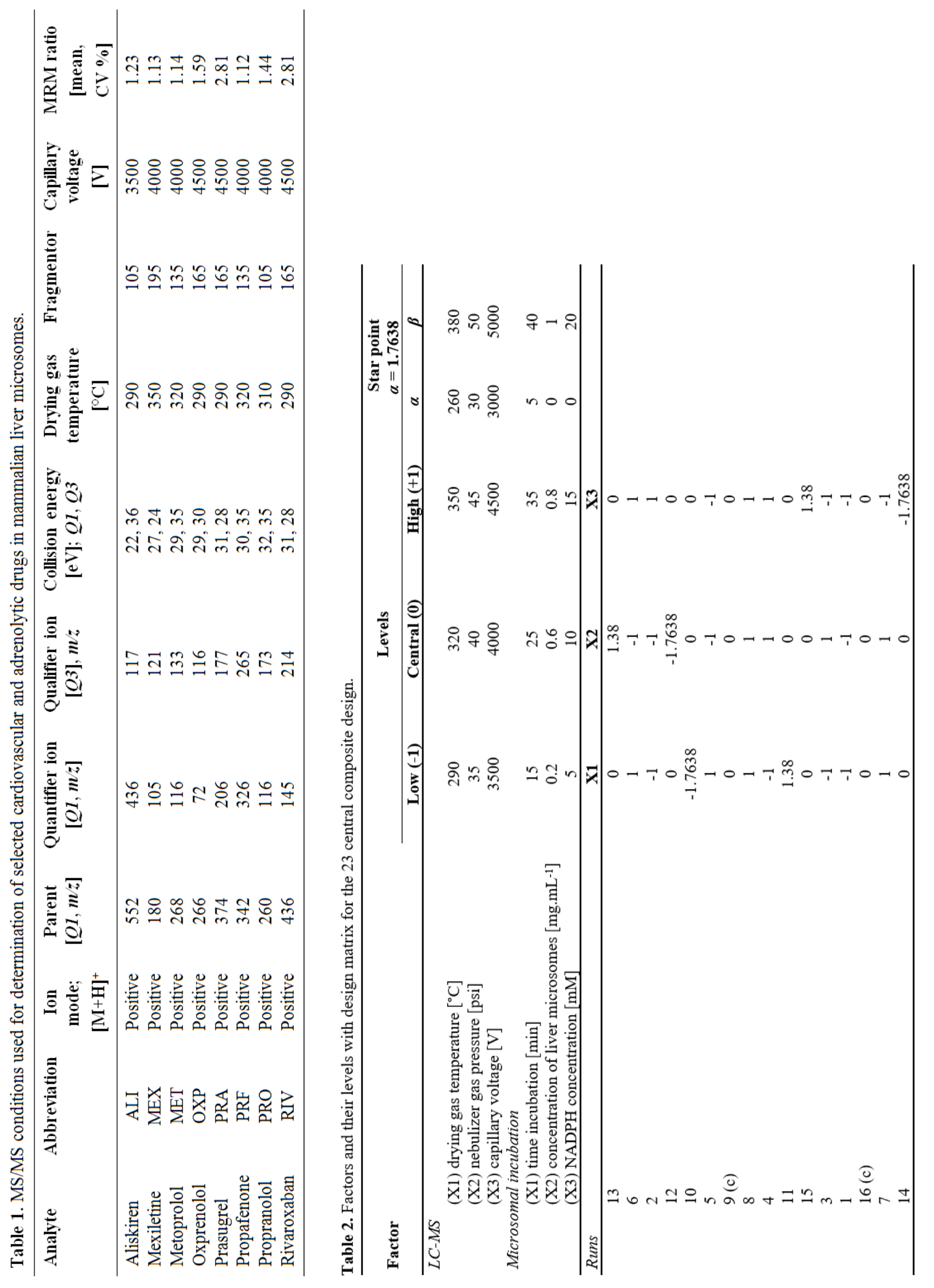


using a syringe pump (Table 1). The studied target compounds were identified by MRM transitions of the compounds searched for using a dwell time of $100 \mathrm{~ms}$ and product ion scan MS/MS experiments. The MS/MS conditions were optimized as well. All the compounds were tested in both positive and negative ion modes. MS/MS parameters were optimized in a continuous flow mode using an integrated syringe pump at a flow rate $5 \mu \mathrm{L} \cdot \mathrm{min}^{-1}$ of standard solutions. The nebulizer gas and dry gas was nitrogen, and helium (99.999 \%) was used as the collision gas in the ion trap. After the best conditions for isolating the precursor on (proton adduct of the analyte) had been determined, full scan MS/MS mode was used to record product ions from the standard solution of each target compound. For each analyte, the fragmentation amplitude and isolation width were also optimized manually to increase the selectivity and sensitivity of the method and to select the most intensive and characteristic fragmentation ions for a qualitative analysis and one of the highest intensity for the quantitative analysis.

\section{Application to patient samples}

A $100 \mu \mathrm{L}$ volume of plasma was transferred to a disposable $2 \mathrm{~mL}$ centrifuge tube. Subsequently, $350 \mu \mathrm{L}$ of water and $150 \mu \mathrm{L}$ of methanol were added, and the tube contents were then mixed gently. $900 \mathrm{~mL}$ of acetonitrile was added to this solution, and the sample was mixed vigorously for few seconds. After a standing time of $15 \mathrm{~min}$, the mixture was centrifuged at 5,000 rpm for $5 \mathrm{~min}$, and $100 \mu \mathrm{L}$ of the clear supernatant was mixed with $900 \mathrm{~mL}$ of $10 \%$ methanol.

\section{Results and Discussion}

\section{Optimization of the LC-MS/MS parameters}

The determination of analytes with a mass spectrometer was carried out in positive polarization (ESI+) in the following modes: full

Table 3. Analysis of variance (ANOVA) for fit of conversion efficiency from central composite design (for PRO).

\begin{tabular}{|c|c|c|c|c|c|}
\hline Source of variation & Sum of square & $\mathrm{Df}^{\mathrm{a}}$ & Mean square & $F$-value ${ }^{\text {b }}$ & $P$-value \\
\hline$\overline{X_{1}}$ & 6183693 & 1 & 6183693 & 25.60267 & 0.002311 \\
\hline $\mathrm{X}_{1}^{2}$ & 1471186 & 1 & 1471186 & 6.09123 & 0.048587 \\
\hline $\mathrm{X}_{2}$ & 1024 & 1 & 1024 & 0.00424 & 0.950191 \\
\hline $\mathrm{X}_{2}^{2}$ & 832855 & 1 & 832855 & 3.44831 & 0.112697 \\
\hline $\mathrm{X}_{3}$ & 2744578 & 1 & 2744578 & 11.36352 & 0.015024 \\
\hline $\mathrm{X}_{3}^{2}$ & 490002 & 1 & 490002 & 2.02878 & 0.204213 \\
\hline $\mathrm{X}_{1} \mathrm{X}_{2}$ & 3583529 & 1 & 3583529 & 14.83707 & 0.008441 \\
\hline $\mathrm{X}_{1} \mathrm{X}_{3}$ & 8270401 & 1 & 8270401 & 34.24238 & 0.001099 \\
\hline $\mathrm{X}_{2} \mathrm{X}_{3}$ & 625918 & 1 & 625918 & 2.59152 & 0.158563 \\
\hline Lack of fit & 1449152 & 6 & 241525 & & \\
\hline Total SS & 38000000 & 15 & & & \\
\hline
\end{tabular}

${ }^{a}$ Df, degrees of freedom;

b Test for comparing model variance with residual (error) variance.

scan (FS), single ion monitoring (SIM), product ion scan (PI), and multiple reaction monitoring (MRM). Scanning in the full mode was performed to determine the $\mathrm{m} / \mathrm{z}$ ratio value of the pseudomolecular ion (an analyte molecule with its mass enlarged by the mass of the attached proton). Single ion monitoring mode was applied to allow us to select the voltage at the fragmentor (the voltage at the end of the capillary directing ions to the analyzer Q1). Product ion mode was to make it possible to obtain product ions for each parent ion through its fragmentation. This step was to use the previously chosen fragmentor voltage, and appropriate values of collision energy were selected for individual studied compounds.

MS/MS transitions form the precursor ion to two of its main fragment ions were registered for each target compound. Only one MRM transition was selected for each at least. Selected MRM transitions for each analyte and the optimal instrumental conditions set for their analysis are summarized in Table 1. 


\section{Central composite design}

Statistica software designed 16 experiments in accordance with the CCD; the levels examined and the experimental results are listed in Table 2. The analysis of variance (ANOVA) was used to assess the significance of each factor and interaction term. The significance of each coefficient was checked using an $F$ test and by determining the $p$ value. The $p$ value was used to check the significance of each coefficient, which indicated the strength of the interaction between each independent variable. ANOVA of the quadratic regression model showed that the model was significant $(p<0.0001 / 0.0005)$ and that the lack-of-fit was not significant
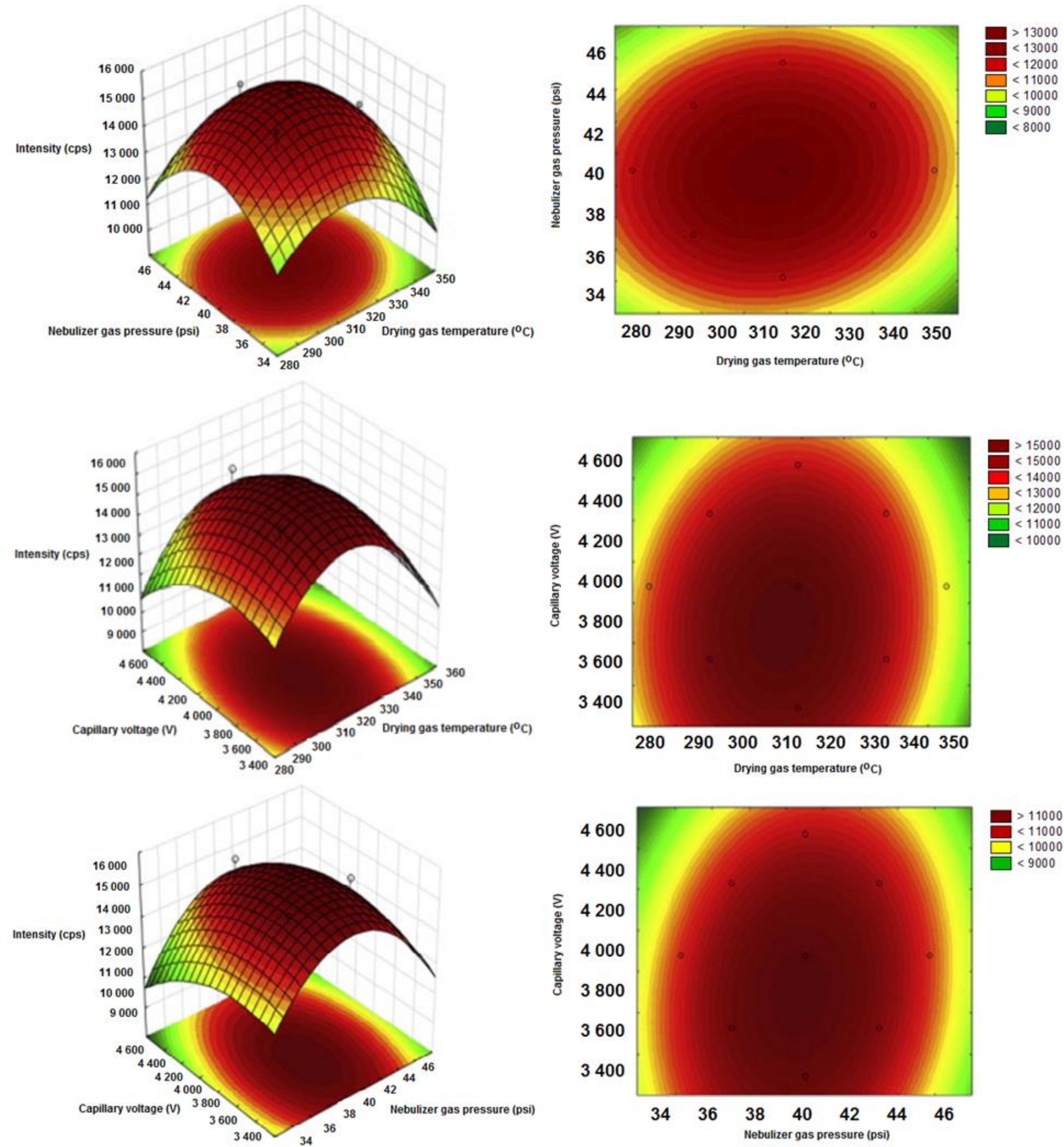

Fig. 1. Three-dimensional surface for the LC-MS analyses using Box-Wilson procedure obtained by plotting. Response surface plots showing the effects of drying gas temperature, nebulizer gas pressure and capillary voltage on the intensity of PRO. 
$(p>0.05)$, indicating that the quadratic model was valid for this study (Table 3 ). The coefficient of determination $\left(R^{2}\right)$ is a measure of the global fit of the model. In our analyses, $R^{2}$ was higher than 0.9860 for all the target compounds, which means that the obtained model could explain the variability in response. Based on the experimental data, a second-order polynominal model was constructed by $\mathrm{CCD}$; where $Y$ is the predicted response value, and $X_{1}, X_{2}$, and $X_{3}$ are the coded values of drying gas temperature, nebulizer gas pressure and capillary voltage, respectively.

In the optimum design process, response surface curves were not only plotted to investigate the relationship between responses and each variable and the interactions of two variables, but the optimal level of each variable was also determined for the maximum response. The typical three-dimensional response surface plots for PRO are shown in Fig. 1. As can be seen, drying gas temperature, nebulizer gas pressure and capillary voltage had quadratic effects on the PRO response. Based on the plotted surfaces, the response value reached a maximum when drying gas temperature, nebulizer gas pressure and capillary voltage was around the central points. After the results had been analyzed, the HPLC-MS analysis conditions $\left(X_{1}=310{ }^{\circ} \mathrm{C}, X_{2}=40 \mathrm{psi}, X_{3}=4,000 \mathrm{~V}\right.$ for PRO $)$ were obtained.

Fig. 1a illustrates the effects of drying gas temperature and nebulizer gas pressure (psi) on the signal intensity during LC-MS analyses. The maximum value was obtained with the drying gas temperature located between 300 and $330{ }^{\circ} \mathrm{C}$ and nebulizer gas pressure (psi) located between 37 and 43. Higher drying gas temperature and higher nebulizer gas pressure tended to result in a decrease of signal intensity. This also could be due to the fact that the decreasing intensities result from ion suppression effects in the electrospray. Fig. 1b shows the effects of drying gas temperature and capillary voltage on the signal intensity during LC-MS analyses. The maximum efficiency could be obtained with $310{ }^{\circ} \mathrm{C}$ and capillary voltage equaling 4,000 V. This drying gas temperature and capillary voltage resulted in the increase in the signal intensity during LC-MS analyses. In the range of $300-330{ }^{\circ} \mathrm{C}$ signal intensity increased, while above the value of $330{ }^{\circ} \mathrm{C}$ no increase in the intensity was observed. A higher drying gas temperature and capillary voltage resulted in medium signal intensity, which would result in a small number of ions to the mass spectrum originating from precursor ion.

Fig. 1c shows the effects of nebulizer gas pressure (psi) and capillary voltage on the signal intensity during LC-MS analyses. It was observed that in the nebulizer gas pressure range of $38-42 \mathrm{psi}$ and in capillary voltage range of 3,600 - 4,200 V signal intensity increased, while above those values no incrementation was observed. The maximum predicted value of signal intensity during LC-MS analyses was under the conditions presented in Table 1.

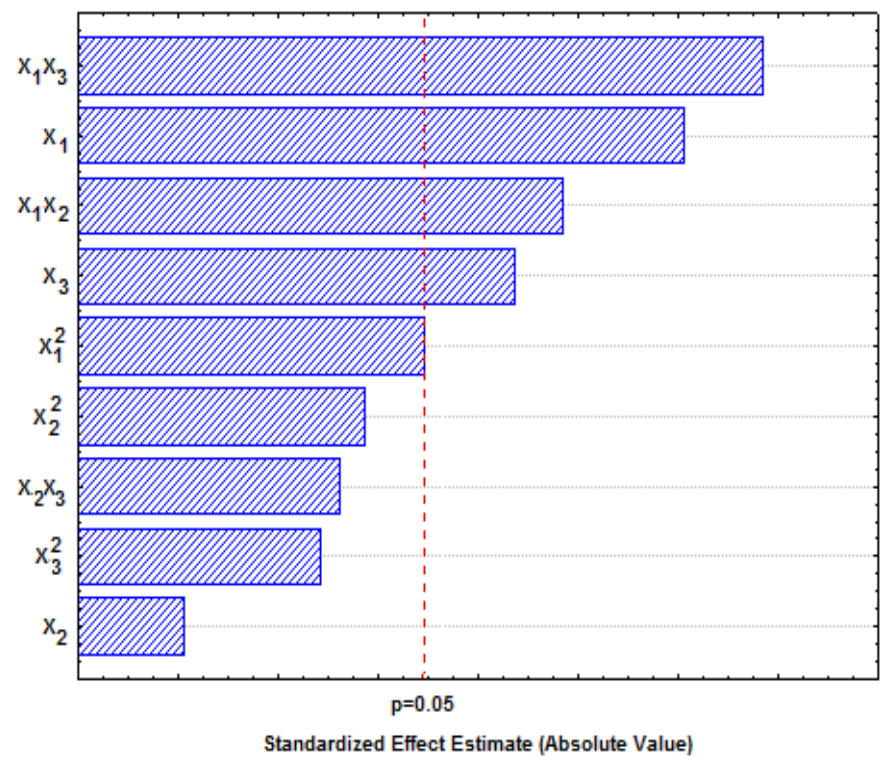

Fig. 2. Pareto chart of the standardized main effect for the CCD design for LC-MS method. The vertical line defines the $95 \%$ confidence level.

The plot of experimental values of efficiency of conversion versus those calculated from equation indicated a good fit, as presented in Fig. 2 (for PRO). As result of the full factorial design, Pareto chart was drawn for each studied cardiovascular drug in order to visualize the estimated effects of the main variables and their interactions. Pareto chart gives a graphical presentation for these effects and it allows looking at both the magnitude and the importance of an effect. In the above mentioned Pareto charts, the bars (variables) that graphically overpass the significance line exert a statistically significant influence on the results obtained. 
In vitro incubation of selected cardiovascular and adrenolytic drugs with mammalian liver microsomes

There are a large number of components in the HLM, among which many compounds may elute in the same time with the target compounds during the analysis. In order to solve this problem, we performed using a Mass Hunter Metabolite ID software; peaks present in the analyte were evaluated in relation to control samples by comparing their retention time in postacquisition analyses, MS spectra and peak area. In order to identify the potential metabolites of selected cardiovascular and adrenolytic drugs, their possible structures were assumed according to the known common metabolic pathways and the structures of parent drugs. Then the total ion chromatogram (TIC) of the liver microsomal solution after incubation with a relevant drug was compared with that of blank liver microsomal solution (without NADPH) to find the possible metabolites. Finally, the potential metabolites were identified by comparing their LC-MS and MS2 spectra with those of target compounds.

In the case of RIV 6 metabolites were found and identified in all in vitro incubations. Metabolite structures were first elucidated by liquid chromatography-mass spectrometry with a detailed analysis of the changes in molecular mass and interpretation of the fragmentation patterns, compared with those of the parent drug. The product ion spectra of metabolites M1, M2, M3, M5 and M6 exhibited losses of $18 \mathrm{amu}$ corresponding to $\mathrm{H}_{2} \mathrm{O}$ molecule, 46 amu $(\mathrm{HCOOH}), 58 \mathrm{amu}(\mathrm{HOCCOH})$ and $72 \mathrm{amu}$ $\left(\mathrm{CO}_{2}+\mathrm{CO}\right)$, indicating that metabolism occurred at the morpholinone moiety. The hydroxylated metabolite M4 showed a product ion spectrum that was different from that for the other hydroxylated metabolites, namely M1, M2 and M3. In the case of M4, fragmentation at the oxazolidinone moiety indicated that the hydroxylation had taken place at the oxazolidinone ring. Hydroxylation occurred at the morpholinone moiety, leading to metabolites M1, M2 and M3 and at the oxazolidinone moiety, leading to metabolite 4. In the case of M5 and M6, combinations of morpholinone and oxazolidinone hydroxylation were also identified. Among all oxidative reactions, the hydroxylation leading to M1 was the major primary pathway identified in incubations with liver microsomes across all the species.

In the case of PRA 4 metabolites were found and identified in all in vitro incubations. One of them was characterized for thiolactone metabolite M1 (in the literature named as R-95913). Then thiolactone metabolite was oxidatively metabolized by hepatic cytochrome P450 to the compound M2 containing a thiol group (in the literature named as R-138727), which was further metabolized to its S-methyl analogue M3 (in the literature named as R-106583). The analytes were analyzed by an LC-MS/MS in the multiple reaction monitoring mode using the respective $[\mathrm{M}+\mathrm{H}]+$ ions, $m / z, 332-109$ for $\mathrm{M} 1, m / z, 498-206$ for M2, $m / z, 364-316$ for M3 and $m / z, 390-318$ for M4.

In the case of ALI 4 metabolites were found and identified in all in vitro incubations. The mass spectra of metabolites M1 and M2 showed molecular ions at $\mathrm{m} / \mathrm{z} 538$, indicating that they are demethylated metabolites. $O$-demethylation was assigned to the region of fragment $\mathrm{C}$ in the two possible positions for methoxy groups. The mass spectrum of metabolite M3 showed a molecular ion at $m / z$ 480. $O$-dealkylation was assigned to the region of fragment $\mathrm{C}$, indicating oxidation of ALI, with loss of the propoxy side chain. Hence, compound M3 could be the methoxy phenol derivative. Metabolite M4 was identified as a glucuronic acid conjugate of M3.

In the case of PRO 7 metabolites were found and identified in all in vitro incubations. This molecule is interesting because of the availability of numerous oxidation sites present on the structure. Hence, the first step was to identify hydroxylated metabolites. The presence of monohydroxylated $(\mathrm{m} / z$ at 276) and dihydroxylated propranolol $(\mathrm{m} / \mathrm{z}$ at 292) metabolites was observed. The abundance of $\mathrm{m} / \mathrm{z}$ 260, the parent ion, was low, demonstrating that the drug was extensively metabolized. During the study also the presence of hydroxypropranolol glucuronide $(\mathrm{m} / \mathrm{z} \quad 452)$ and dihydroxypropranolol glucuronide $(\mathrm{m} / \mathrm{z}, 468)$ was observed.

In the case of OXP 2 metabolites were found and identified in all in vitro incubations. The proposed microsomal transformation 


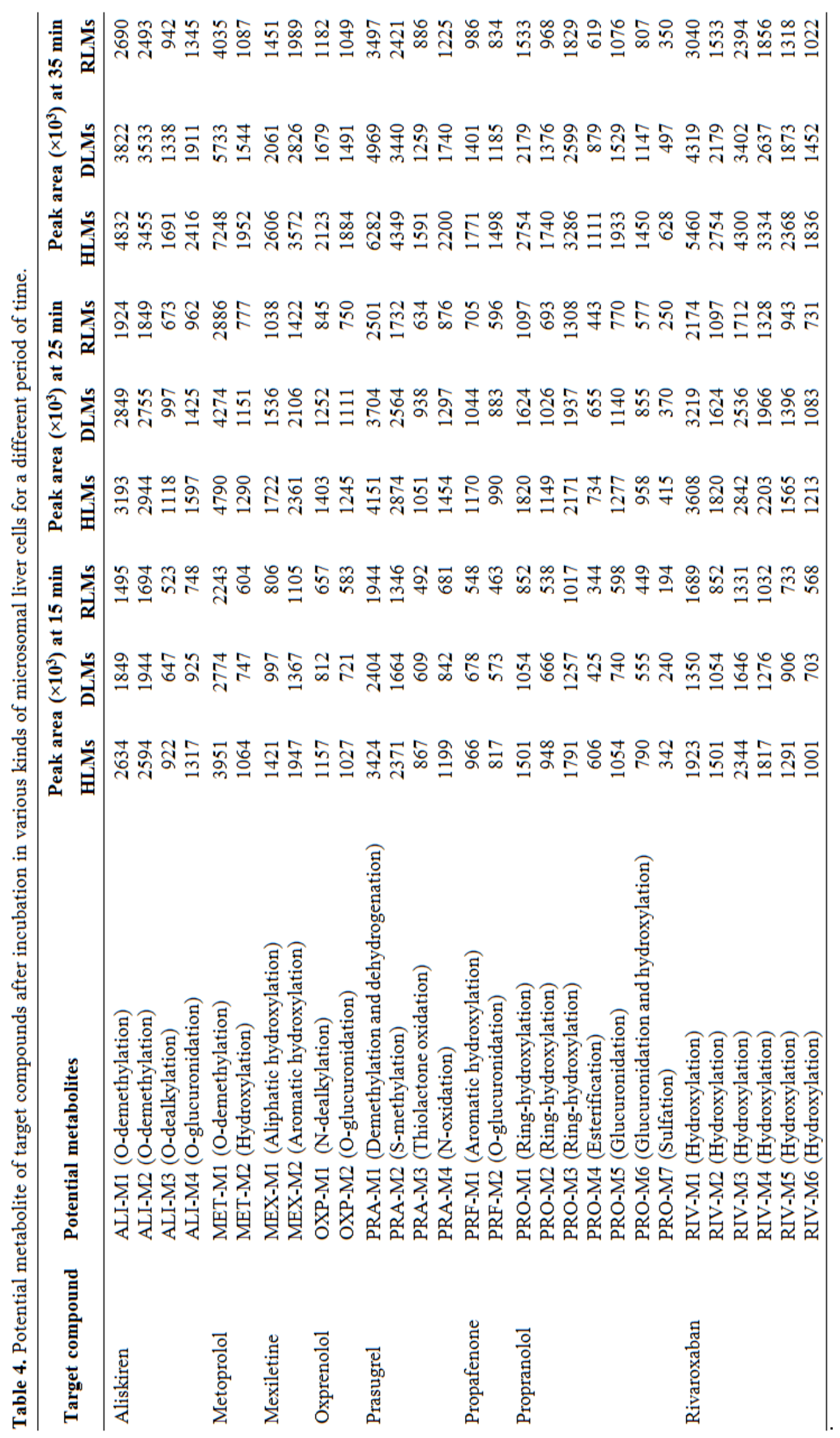


of oxprenolol $(\mathrm{m} / \mathrm{z}, 224)$ include $N$-dealkylation and ring-hydroxylation (O gain) as well as the combination of these two pathways. During the study also the presence of hydroxyoxprenolol glucuronide $(\mathrm{m} / \mathrm{z}$ 571) was observed.

In the case of MET 2 metabolites were found and identified in all in vitro incubations. The proposed microsomal conversion of metoprolol include benzylic hydroxylation or aromatic hydroxylations $(\mathrm{m} / \mathrm{z} 284)$ and demethylation $(\mathrm{m} / \mathrm{z} 254)$.

In the case of MEX 2 metabolites were found and identified in all in vitro incubations. The proposed microsomal conversion of mexiletine include aliphatic $(\mathrm{m} / \mathrm{z}, 178)$ and aromatic $(\mathrm{m} / \mathrm{z}, 196)$ hydroxylation.

In the case of PRF 2 metabolites were found and identified in all in vitro incubations. For propafenone $(\mathrm{m} / \mathrm{z}$ 342) with a known metabolism, the only product characterized for phase I that was observed and identified was single $\mathrm{O}$ gain at various positions. In other words, the only metabolic pathways that was mimicked with the use of microsomal fraction was the hydroxylation $(\mathrm{m} / \mathrm{z}, 358)$. During the study also the presence of hydroxypropafenone glucuronide $(\mathrm{m} / \mathrm{z}, 647)$ was observed.

To elucidate the structures of the unknown metabolites, we studied the fragmentation of the $[\mathrm{M}+\mathrm{H}]+$ ions of MET and its metabolites using LC/ESI/MS/MS experiments. Major metabolites were compared with the retention time of the commercial reference standards. The postulated major metabolic pathways of incubated cardiovascular and adrenolytic drugs with DLMs, HLMs and RLMs is presented in Table 4. These pathways show that the hydroxyl moiety, carbon-carbon double bond, and carbonoxygen double bond are the metabolic sites in vitro. The hydroxyl groups is the primary metabolic sites with further formation through reduction and oxidation to generate dehydrated and hydrogenated metabolites, which is similar to the investigated in vivo metabolism in real biological samples. The production of potential metabolites of target compounds was all increased with the prolongation of the incubation time. Among them, the demethylated metabolites were the major metabolites, while the hydroxylated were the minor metabolites; glucuronidated constituted the fewest of target compounds. Based on the above discussion, the selected chromatograms of ALI in 3 kinds of liver is presented in Fig. 3. This may be due to the different genotype and activity of hepatic metabolic enzymes in different species. The detailed proposed fragmentation for all potential metabolites is presented in Table 5.
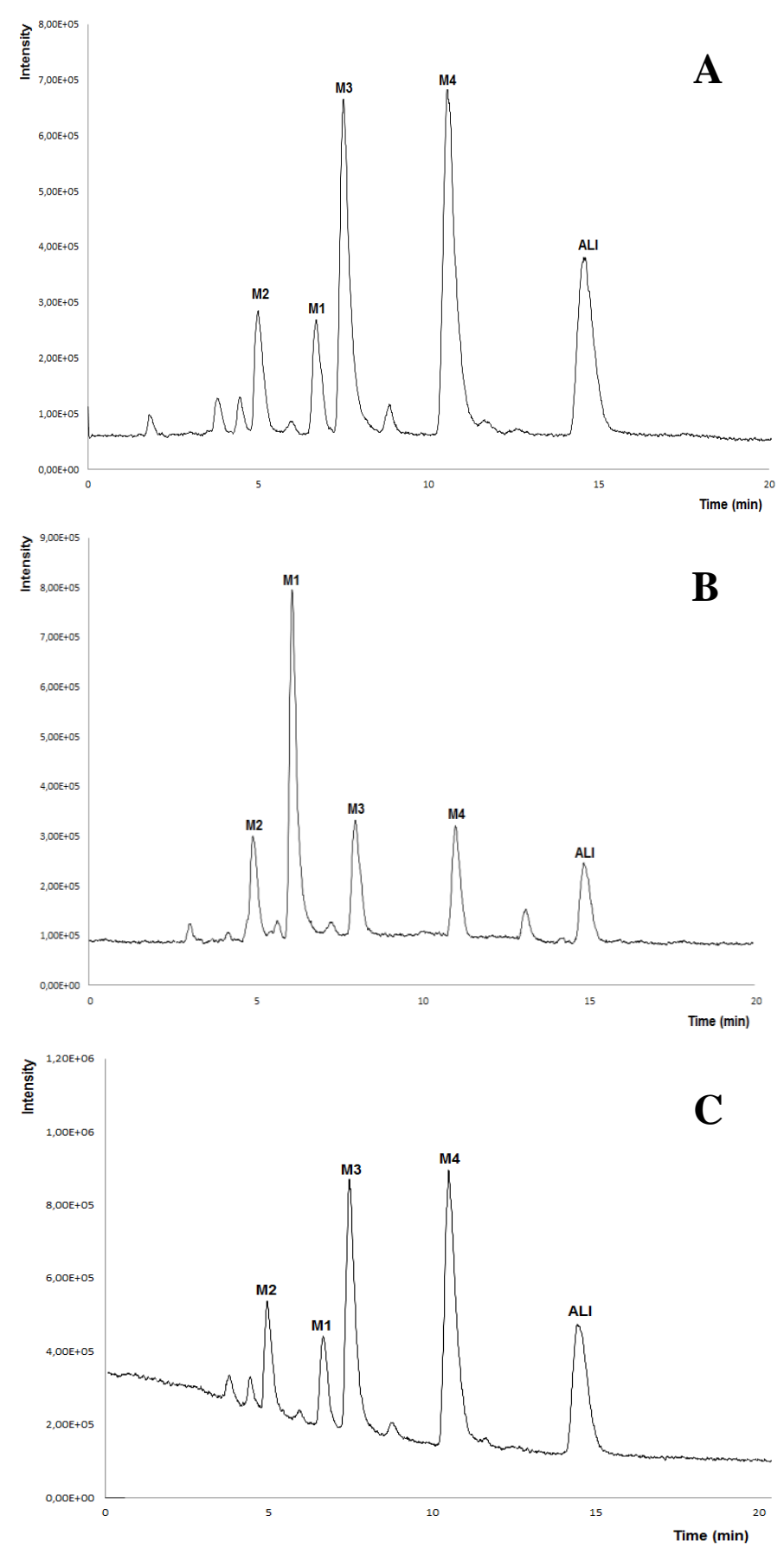

Fig. 3. HPLC-chromatograms of ALI potential metabolites (M1 - M4) in three types liver microsomes: (A) DLMs, (B) HLMs and (c) RLMs.

All the postulated phase I and II metabolic reactions are typical of processes catalyzed 


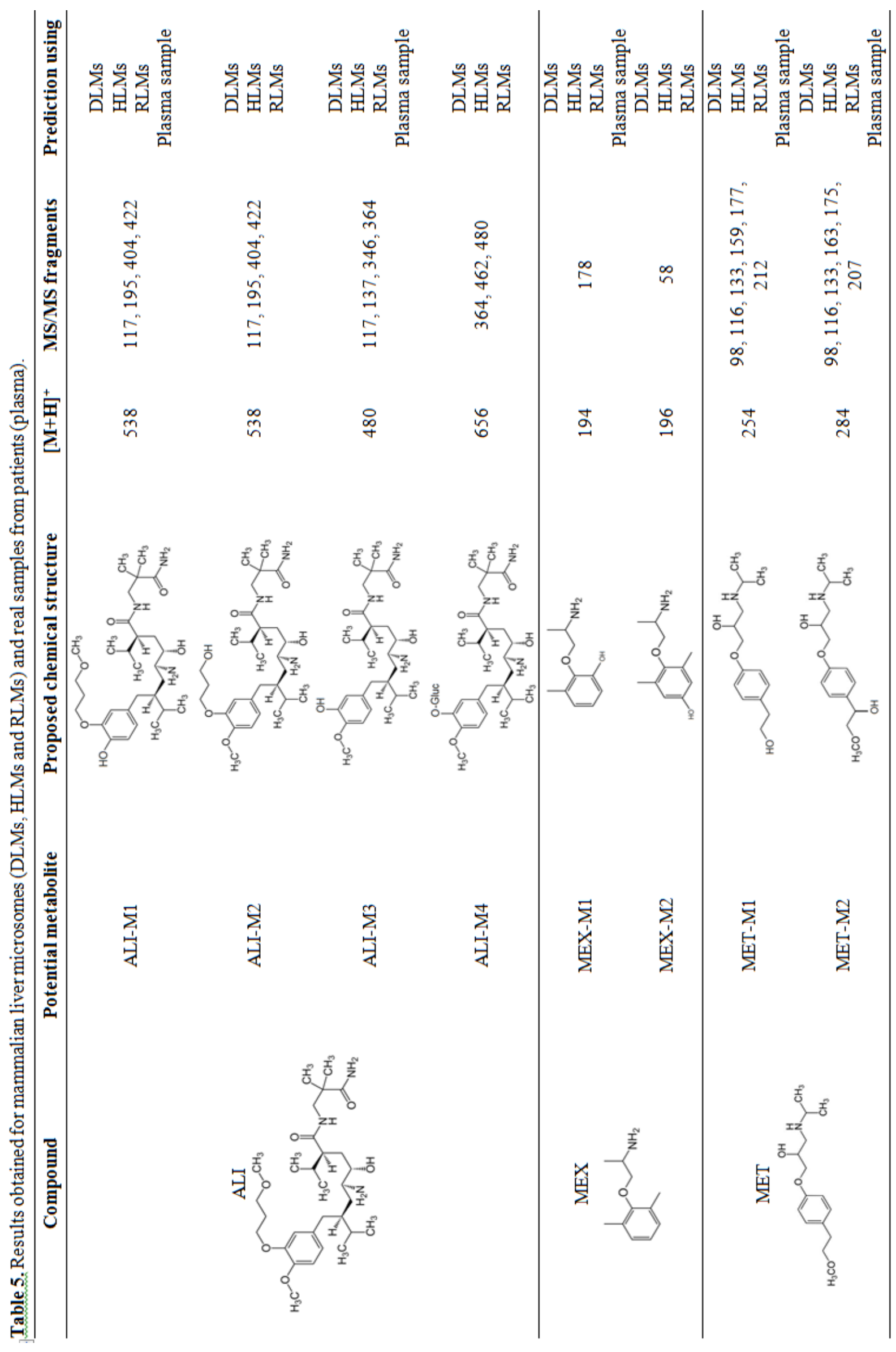




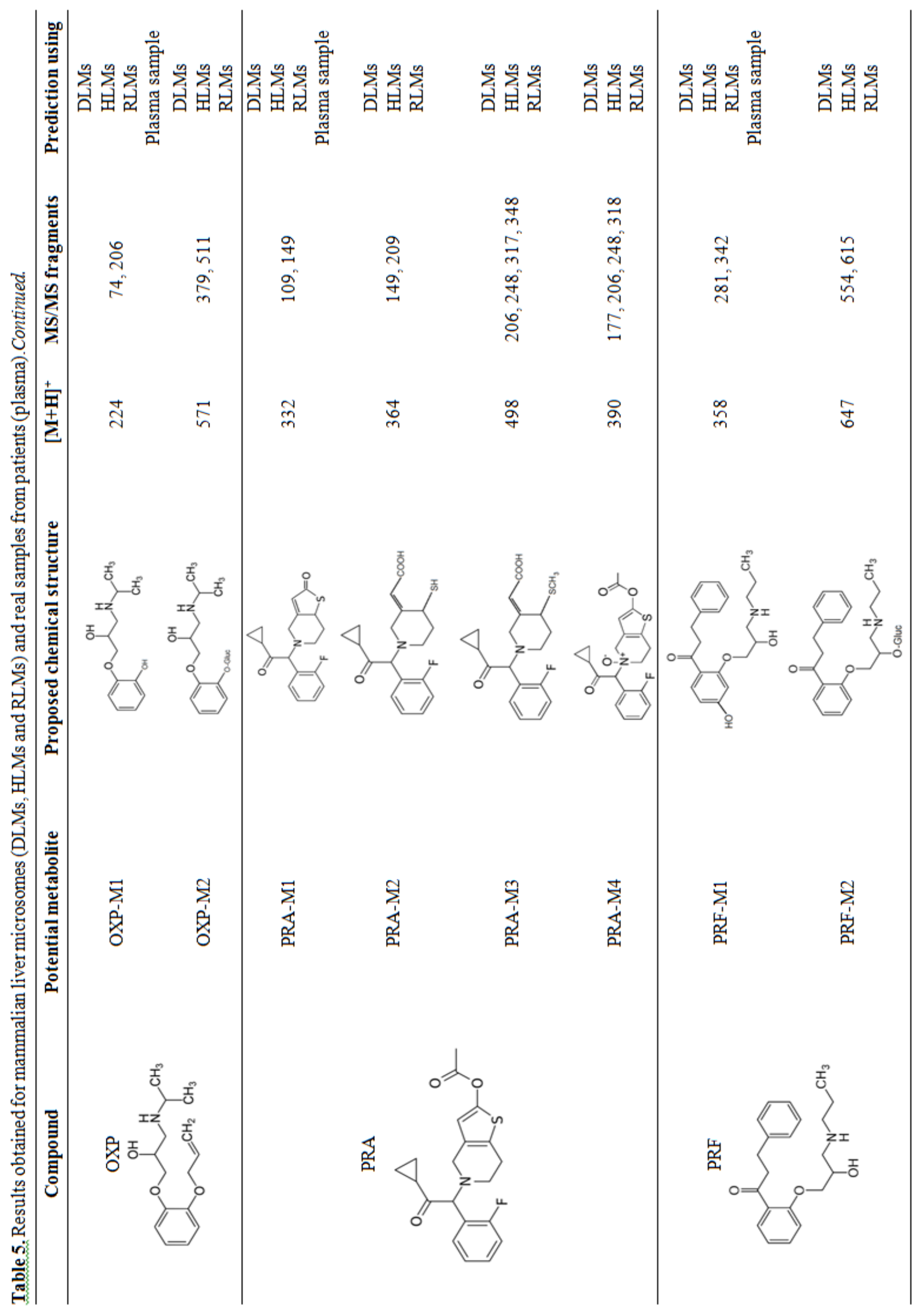




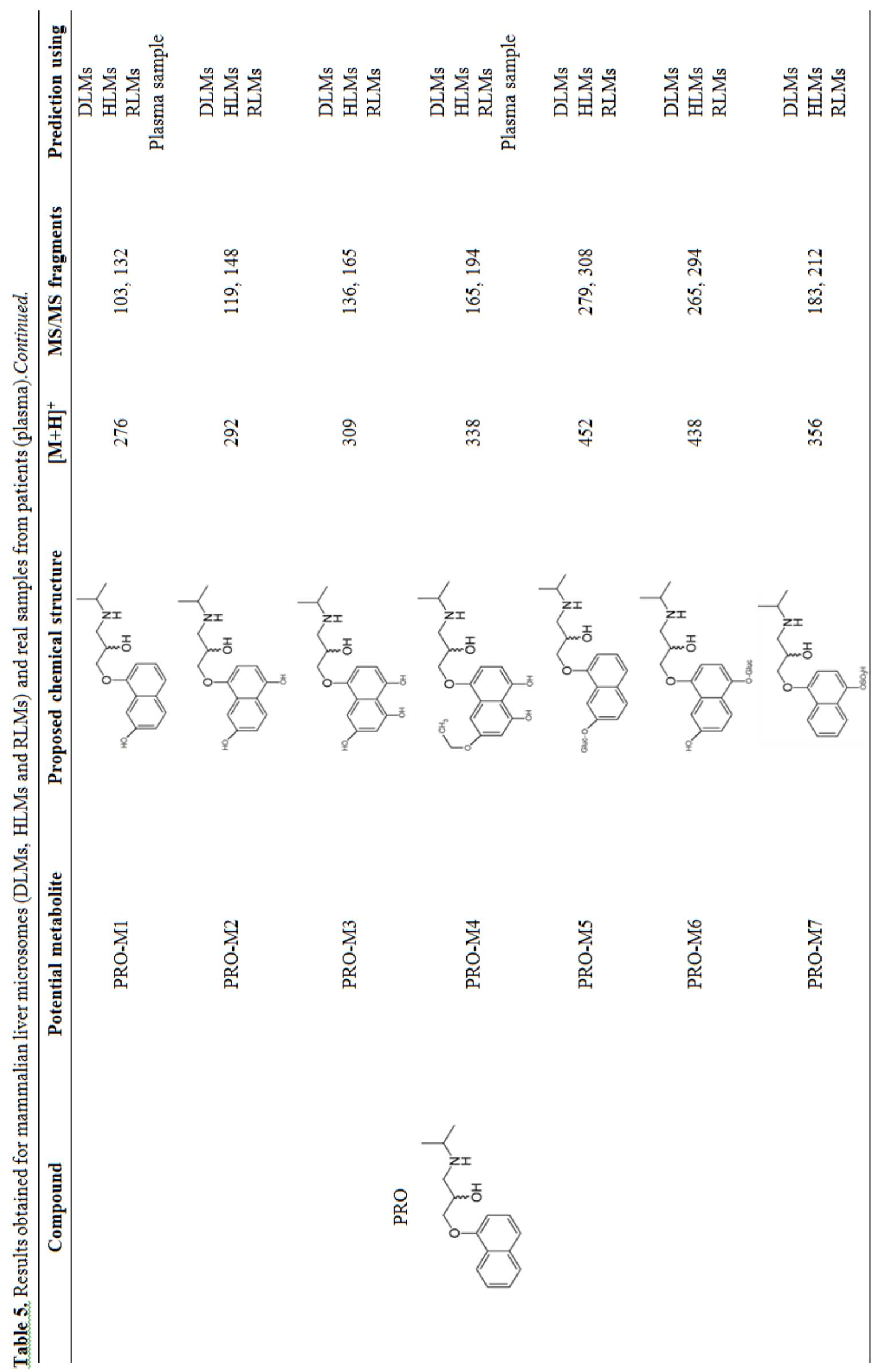




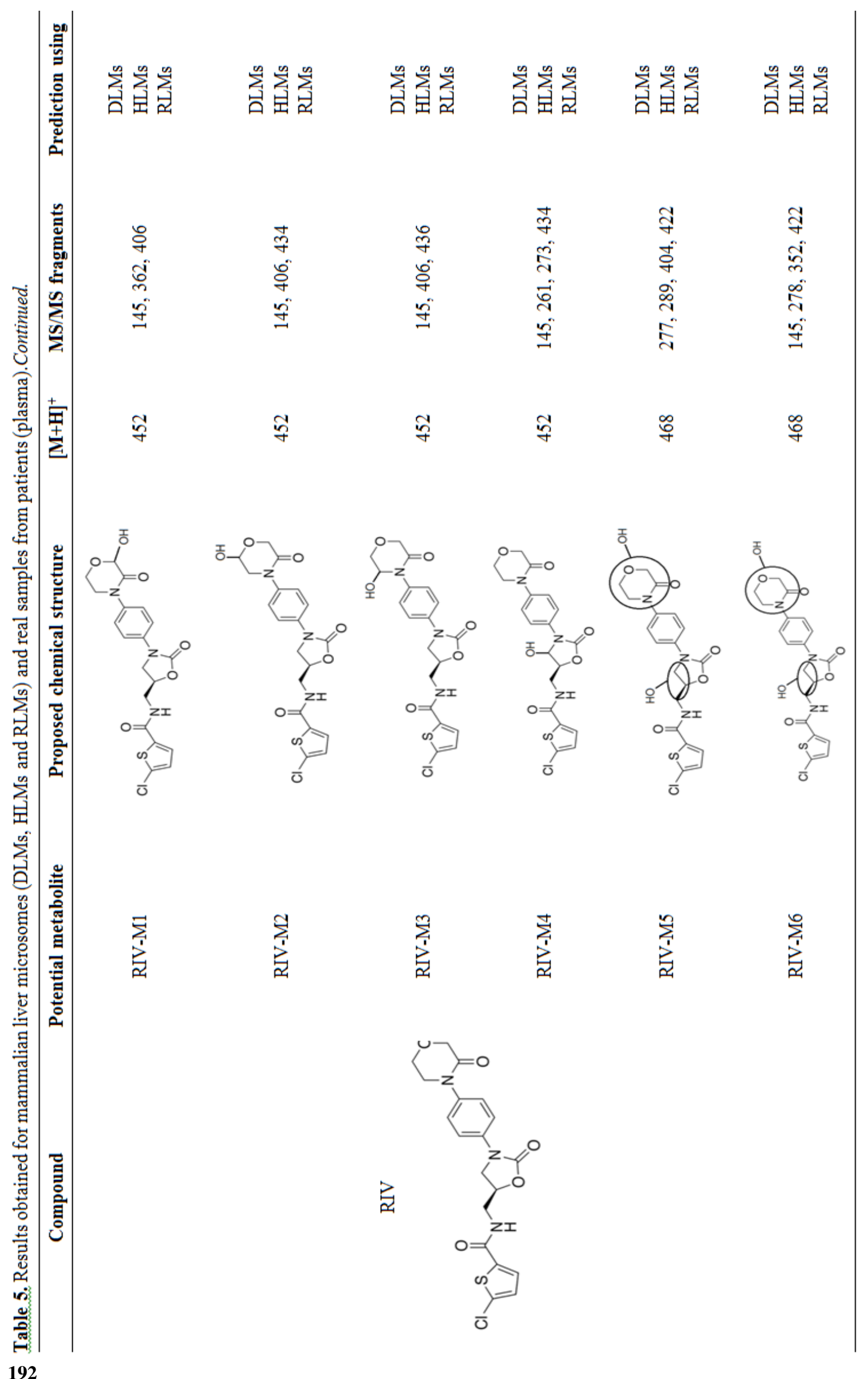


by isoenzymes of the cytochrome P450 group. It may be concluded that the mammalian liver microsomes having enzymatic activity may be very useful in the study of the metabolism of cardiovascular and adrenolytic drugs.

\section{Analysis of biological samples from patients}

In order to examine the proposed metabolic pathways of target compounds, the data coming from the suggested method with the use of liver microsomes fractions were studied in detail and were compared with the results obtained from biological samples originating from patients receiving cardiovascular and adrenolytic drugs. The presence of drug and its potential metabolites in samples from the patients were additionally confirmed by MS/MS spectra. The abundance of relevant parent ion, was low, demonstrating that the drug was extensively metabolized. Applying the developed LC-MS/MS method in plasma samples were identified: metoprolol and its two metabolites $O$-demethylated metoprolol $(\mathrm{m} / z=254)$ and hydroxylated metoprolol $(\mathrm{m} / \mathrm{z}=284)$; propranolol and hydroxylated propranolol $(\mathrm{m} / \mathrm{z}=276)$ was observed; mexiletine and hydroxylated mexiletine $(\mathrm{m} / z=196)$; oxprenolol $\mathrm{N}$-dealkylated oxprenolol $(\mathrm{m} / \mathrm{z}=224)$ and propafenone and hydroxylated propafenone $(m / z=358)$, respectively.

\section{Conclusions}

In this study, we applied LC-MS/MS to identify phase I and phase II metabolites of selected cardiovascular and adrenolytic drugs as a model compounds detected after in vitro incubation of the studied drug with dog, human and rat liver microsomes fraction. To the best of our knowledge, no detailed information regarding the structure of their potential metabolites via in vitro studies has been published. The results obtained show the similarity among analyzed mammalian species. The main biotransformation route of the studied compounds was identified as reduction, oxidation, dehydroxylation and glucuronic acid conjugation. As a result, the presented approach with in vitro and in vivo studies can be helpful in prognosing the influence of the selected drugs and their metabolites from different therapeutic groups on the activity of metabolizing enzymes; it may also be used in planning a targeted therapy.

\section{Acknowledgments}

The work was financially supported by the National Science Centre in the frame of the project Sonata 12, No. 2016/23/D/ST4/00305 (2017-2020).

\section{Conflict of Interest}

The authors declare that they have no conflict of interest.

\section{References}

Bussiere JL (2008) Species selection consideration for preclinical toxicology studies for biotherapeutics. Expert Opin. Drug Metab. Toxicol. 4: 871-877.

Cholerton S, Daly AK, Idle JR (1992) The role of individual human cytochromes P450 in drug metabolism and clinical response. Trends Pharmacol. Sci. 13: 434-439.

Degtyarenko KN, Archakov AI (1993) Molecular evolution of P450 superfamily and P450-containing monooxygenase systems. FEBS Lett. 11: 1-8.

Goldman DP, Joyce GF, Escarce JJ, Pace JE, Solomon MD, Laouri M, Landsman PB, Teutsch SM (2004) Pharmacy benefits and the use of drugs by the chronically ill. JAMA 291: 2344-2350.

Guengerich FP (2000) Common and uncommon cytochrome $\mathrm{P} 450$ reactions related to metabolism and chemical toxicity. Chem. Res. Toxicol. 14: 611-650.

Houston JB (1994) Utility of in vitro drug metabolism data in predicting in vivo metabolic clearance. Biochem. Pharm. 47: 1469-1479.

Ingelman-Sundberg M (2004) Pharmacogenetics of cytochrome $\mathrm{P} 450$ and its applications in drug therapy: the past, present and future. Trends Pharmacol. Sci. 25: 193-200.

Ingelman-Sundberg M, Oscarson M, McLellan RA (1999) Polymorphic human cytochrome P450 enzymes: an opportunity for individualized drug treatment. Trends Pharmacol. Sci. 20: 342-349.

Isin EM, Guengerich FP (2007) Complex reactions catalyzed by cytochrome P450 enzymes. Biochim. Biophys. Acta 1770: 314-329.

Jia L, Liu X (2007) The conduct of drug metabolism studies considered good practice (II): in vitro experiments. Curr. Drug Metab. 8: 822-829.

Kang JS, Lee MH (2009) Overview of therapeutic drug monitoring. Korean J. Intern. Med. 24: 1-10.

Lamb DC, Waterman MR, Kelly SL, Guengerich FP (2007) Cytochromes P450 and drug discovery. Curr. Opin. Biotechnol. 18: 504-512.

Li AP (2001) Screening for human ADME/Tox drug properties in drug discovery. Drug Discov. Today 6: 357-366. 
Lietz CB, Gemperline E, Lingjun L (2013) Qualitative and quantitative mass spectrometry imaging of drugs and metabolites. Adv. Drug Delivery Rev. 65: 1074-1085.

Nicholson JK, Lindon JC, Holmes E (1999) 'Metabonomics': understanding the metabolic responses of living systems to pathophysiological stimuli via multivariate statistical analysis of biological NMR spectroscopic data. Xenobiotica 29: 1181-1189.

Persell SD (2011) Prevalence of resistant hypertension in the United States, 2003-2008. Hypertension 57: 10761080.

Rostami-Hodjegan A, Tucker GT (2007) Simulation and prediction of in vivo drug metabolism in human populations from in vitro data. Nat. Rev. Drug Discov. 6: 140-148.

Sahi J, Grepper S, Smith C (2010) Hepatocytes as a tool in drug metabolism, transport and safety evaluations in drug discovery. Curr. Drug Discov. Technol. 7: 188-198.

Wicha SG, Kloft Ch (2018) Quantitative systems pharmacology in model-informed drug development and therapeutic use. Curr. Opin. Sys. Biol. 10: 19-25.

Wienkers LC, Heath TG (2005) Predicting in vivo drug interactions from in vitro drug discovery data. Nat. Rev. Drug Discov. 4: 825-833.

Wrighton SA, Ring BJ, VandenBranden M (1995) The use of in vitro metabolism techniques in the planning and interpretation of drug safety studies. Toxicol. Pathol. 23: 199-208.

Xu RN, Fan L, Rieser MJ, El-Shourbagy TA (2007) Recent advances in high-throughput quantitative bioanalysis by LC-MS/MS. J. Pharm. Biomed. Anal. 44: 342-355.

Yan Z, Caldwell GW (2001) Metabolism profiling, and cytochrome $\mathrm{P} 450$ inhibition \& induction in drug discovery. Curr. Topics Med. Chem. 1: 403-425.

Zhang Z, Tang W (2018) Drug metabolism in drug discovery and development. Acta Pharm. Sin. B 8: 721-732.

Zisaki A, Miskovic L, Hatzimanikatis V (2015) Antihypertensive drugs metabolism: an update to pharmacokinetic profiles and computational approaches. Curr. Pharm. Des. 21: 806-822. 